\title{
Maximal and Willmore Null Hypersurfaces in Generalized Robertson-Walker Spacetimes
}

\author{
Cyriaque Atindogbe $^{1 *}$ and Hippolyte Hounnon ${ }^{1}$ \\ ${ }^{1}$ Department of Mathematics, Faculty of Science and Techniques, University of Abomey-Calavi, Benin \\ * Corresponding author
}

\begin{abstract}
Article Info
Keywords: Generalized RobertsonWalker spacetimes, Maximal null hypersurface, Willmore null hypersurface 2010 AMS: 53C42, 53C50, 53C21

Received: 4 January 2020

Accepted: 25 April 2020

Available online: 10 June 2020
\end{abstract}

\begin{abstract}
We establish after some technical results a characterization of maximal null hypersurfaces in terms of a constant mean curvature screen foliation (in the slices) induced by the Chen's vector field. Thereafter, bounds are provided for both the squared norm of the (screen) shape operator for non totally geodesic maximal null hypersurfaces and the scalar curvature of the fiber. In terms of the scalar curvature of the fiber and the warping function, we establish necessary and sufficient conditions for Null Convergence Condition (NCC) to be satisfied in which case we prove that there are no non totally geodesic maximal null hypersurfaces. A generic example consisting of graphs of functions defined on the fiber is given to support our results. Finally, we provide lower bounds for the extrinsic scalar curvature and give a characterization result for Willmore null hypersurfaces in generalized Robertson-Walker spacetimes.
\end{abstract}

\section{Introduction}

The study of maximal spacelike hypersurfaces in Lorentzian manifolds is an important topic as evidenced by the considerable amount of papers devoted to this purpose ([1]-[13], and references therein). The big amount of interest to these objects is due to to the fact that they play a key role in the dynamic aspects of general relativity and are solutions of existence and uniqueness Calabi-Berstein type problems [2,6]. The reason for the terminology maximal (in contrast to the minimality in Riemannian setting ) is that the vanishing of the mean curvature is equivalent to the fact that the hypersurface realizes a local maximum of the area functional for compactly supported normal variation. Willmore hypersurfaces are generalization of the maximal ones. They are critical point of the total squared mean curvature functional whose study was proposed by Willmore in 1965. Most of the works done since then are on (nondegenerate) 2-dimensional surfaces in (semi-)Riemannian setting [14]-[17].

Null hypersurfaces are genuine objects in Lorentzian geometry in the sense that they have not Riemannian counterpart. They are very interesting in general relativity and black hole horizons are one of the most remarkable examples, and recent works show that there is an increasing interest on null hypersurfaces both from a physical and geometrical point of view [18]-[24].

In this paper we are interested in maximal and Willmore null hypersurfaces in generalized Robertson-Walker spacetimes. Our main aim is to give existence and characterization results both for maximal and Willmore null hypersurfaces and bring out some of their geometric properties.

The paper is organized as follows. In Section 2 we revise some facts about null hypersurfaces in Lorentzian manifolds with special attention paid to their connection with Chen's concircular vector field and symmetries of generalized Robertson-Walker 
spacetimes. Section 3, after some technical results (Proposition 3.1), presents a characterization of maximal null hypersurfaces in terms of a constant mean curvature screen foliation induced by Chen's vector field in the slices (Theorem 3.2). Also, necessary and sufficient conditions to obey the Null Convergence Condition (NCC) are provided in terms of the scalar curvature of the fiber and the warping function. In this case, we prove that there are no non totally geodesic maximal null hypersurfaces. Upper and lower bounds are provided for both the squared norm of the (screen) shape operator of non totally geodesic maximal null hypersurfaces and the scalar curvature of the fiber (Theorem 3.4). In Section 4 we support above results by a generic example consisting of graphs of functions defined on the fiber and establish the maximality condition in terms of the Laplacian of the involved functions (Theorem 4.1). Section 5 is concerned with providing a lower bound for the extrinsic scalar curvature for maximal null hypersurfaces (Theorem 5.2). Finally we give in Section 6 a characterization of Willmore null hypersurfaces in generalized Robertson-Walker spacetimes (Theorem 6.3).

\section{Preliminaries}

\subsection{Some symmetries of generalized Robertson-Walker spacetimes}

A Generalized Robertson-Walker spacetime (GRW in short) is the warped product $\bar{M}=-I \times_{f} F$, where $I$ (the base) is an open interval of the real line $\mathbb{R},\left(F, g_{F}\right)$ the fiber is a Riemannian manifold of dimension $n-1$ and $f>0$ is a smooth warping function (or scale factor) defined on $I$. It is then endowed with the Lorentzian metric

$$
\bar{g}=-d t^{2}+f^{2}(t) g_{F}
$$

where $t$ stands for the natural (global) parameter on $\mathbb{R}$. In particular, when the Riemannian fiber $F$ has constant sectional curvature, then $-I \times_{f} F$ is classically called a Robertson-Walker (RW) spacetime, and it is a spatially homogeneous spacetime. Throughout, $\pi_{I}$ (resp. $\pi_{F}$ ) will denote projection on the base space $I$ (resp. on the fiber $F$ ).

Observe that the existence of a globally defined timelike coordinate vector field $\partial_{t}$ makes a GRW time-orientable. The vector field

$$
\zeta=f \partial_{t}
$$

is timelike, closed and conformal. If $\bar{\nabla}$ denotes the Levi-Civita connection of $\bar{M}$, it holds for vector fields $V$ tangent to $\bar{M}$,

$$
\bar{\nabla}_{V} \zeta=f^{\prime}(t) V
$$

The above definition of GRW spacetimes highlights the existence of a spacelike hypersurface foliation with leaves the slices $\{t\} \times F$ ( spatial universes), $(t \in I)$.

A nice characterization theorem by Chen [25, Theorem 1] states that a Lorentzian manifold of dimension $n \geq 3$ is a GRW spacetime if and only if it admits a timelike concircular vector field. Following Fialkow [26], a concircular vector field is a vector field $v$ which satisfies

$$
\bar{\nabla}_{X} v=\mu X
$$

for vector fields $X$ tangent to $\bar{M}$, where $\bar{\nabla}$ denotes the Levi-Civita connection of $\bar{M}$ and $\mu$ is a smooth function on $\bar{M}$. A vector field $v$ as above is called Chen's vector field. It is an eigenvector of the Ricci tensor of $\bar{M}$ with eigenvalue we denote by $\sigma$. The Weyl tensor of $\bar{M}$ is

$$
W=\bar{R}+\frac{\bar{s}}{2 n(n-1)} \bar{g} \otimes \bar{g}+\frac{1}{n-2}\left(\overline{R i c}-\frac{\bar{s}}{n} \bar{g}\right) \otimes \bar{g}
$$

where we use the following definition for the Ricci tensor : $\overline{\operatorname{Ric}}(X, Y)=\operatorname{trace}(Z \longmapsto \bar{R}(Z, X) Y)$, being $\bar{s}$ the scalar curvature of $\bar{M}$. It can be shown ( [11]) that the components of the Ricci curvature are given by

$$
\bar{R}_{j k}=\frac{n-2}{\langle v, v\rangle} W\left(v, \partial_{j}, v, \partial_{k}\right)+\frac{\bar{s}-\sigma}{n-1}\left(\bar{g}_{j k}-\frac{\left\langle v, \partial_{j}\right\rangle\left\langle v, \partial_{k}\right\rangle}{\langle v, v\rangle}\right)+\sigma \frac{\left\langle v, \partial_{j}\right\rangle\left\langle v, \partial_{k}\right\rangle}{\langle v, v\rangle} .
$$

Using warping coordinate frames, it holds

$$
\begin{aligned}
\overline{\operatorname{Ric}}\left(\partial_{t}, \partial_{t}\right) & =-(n-1) \frac{f^{\prime \prime}}{f} \\
\overline{\operatorname{Ric}}\left(\partial_{t}, X\right) & =0 \\
\overline{\operatorname{Ric}}(X, Y) & =\operatorname{Ric}_{g_{F}}(X, Y)+\left[f f^{\prime \prime}+(n-2) f^{\prime 2}\right] g_{F}(X, Y)
\end{aligned}
$$

where $X$ and $Y$ are tangent to the fiber $F$. 


\subsection{Geometry of null hypersurfaces}

Let $M$ be a null hypersurface in a Lorentzian manifold $\left(\bar{M}^{n}, \bar{g}\right)$, i.e a hypersurface for which the induced metric tensor $g=\bar{g}_{\mid M}$ is degenerate on it. A screen distribution on $M^{n-1}(n \geq 3)$, is a complementary bundle of $T M^{\perp}$ in $T M$. It is then a rank $n-2$ non-degenerate distribution over $M$. In fact, there are infinitely many possibilities of choices for such a distribution. Each of them is canonically isomorphic to the factor vector bundle $T M / T M^{\perp}$. From [20], it is known that for a null hypersurface equipped with a screen distribution, there exists a unique rank 1 vector subbundle $\operatorname{tr}(T M)$ of $T \bar{M}$ over $M$, such that for any non-zero section $\xi$ of $T M^{\perp}$ on a coordinate neighborhood $\mathscr{U} \subset M$, there exists a unique section $N$ of $\operatorname{tr}(T M)$ on $\mathscr{U}$ satisfying

$$
\left.\bar{g}(N, \xi)=1, \quad \bar{g}(N, N)=\bar{g}(N, W)=0,\left.\quad \forall W \in \mathscr{S}(N)\right|_{\mathscr{U}}\right)
$$

where $\mathscr{S}(N)$ denotes the fixed screen distribution.

Then $T \bar{M}$ admits the splitting:

$$
\left.T \bar{M}\right|_{M}=T M \oplus \operatorname{tr}(T M)=\left\{T M^{\perp} \oplus \operatorname{tr}(T M)\right\} \oplus \mathscr{S}(N) .
$$

We call $\operatorname{tr}(T M)$ a (null) transverse vector bundle along $M$. Now, we need to clarify the (general) concept of rigging for null hypersurfaces (see [23] for details).

Definition 2.1. Let $M$ be a null hypersurface in a Lorentzian manifold. A rigging for $M$ is a vector field $\zeta$ defined on some open set containing $M$ such that $\zeta_{p} \notin T_{p} M$ for each $p \in M$.

Given a rigging $\zeta$ in a neighborhood of $M$ in $(\bar{M}, \bar{g})$ let $\alpha$ denote the 1 -form $\bar{g}$-metrically equivalent to $\zeta$, i.e. $\alpha=\bar{g}(\zeta$, .). Take $\omega=i^{\star} \alpha$, being $i: M \hookrightarrow \bar{M}$ the canonical inclusion. Next, consider the tensors

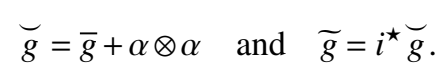

It is easy to show that $\widetilde{g}$ defines a Riemannian metric on the (whole) hypersurface $M$. The rigged vector field of $\zeta$ is the $\widetilde{g}$-metrically equivalent vector field to the 1-form $\omega$ and it is denoted by $\xi$. In fact the rigged vector field $\xi$ is the unique lightlike vector field in $M$ such that $\bar{g}(\zeta, \xi)=1$. Moreover, $\xi$ is $\widetilde{g}$-unitary. To a rigging $\zeta$ for $M$ is associated the screen distribution $\mathscr{S}(\zeta)$ given by $\mathscr{S}(\zeta)=T M \cap \zeta^{\perp}$. It is the $\widetilde{g}$-orthogonal subspace to $\xi$ and the corresponding null transverse vector field on $M$ is

$$
N=\zeta-\frac{1}{2} \bar{g}(\zeta, \zeta) \xi
$$

A null hypersurface $M$ equipped with a rigging $\zeta$ is said to be normalized and is denoted $(M, \zeta)$ (the latter is called a normalization of the null hypersurface). A normalization $(M, \zeta)$ is said to be closed (resp. conformal) if the rigging $\zeta$ is closed i.e the 1-form $\alpha$ is closed (resp. $\zeta$ is a conformal vector field, i.e there exists a function $\rho$ on the domain of $\zeta$ such that $L_{\zeta} \bar{g}=2 \rho \bar{g}$ ). We say that $\zeta$ is a null rigging for $M$ if the restriction of $\zeta$ to the null hypersurface $M$ is a null vector field.

Let $\zeta$ be a rigging for a null hypersurface of a Lorentzian manifold $(\bar{M}, \bar{g})$. The screen distribution $\mathscr{S}(\zeta)=\operatorname{ker} \omega$ is integrable whenever $\omega$ is closed, in particular if the rigging is closed. Throughout, the ambient Lorentzian metric $\bar{g}$ will also be denoted $\langle$,$\rangle .$

On a normalized null hypersurface $(M, \zeta)$, the Gauss and Weingarten formulas are given by

$$
\begin{aligned}
\bar{\nabla}_{X} Y & =\nabla_{X} Y+B(X, Y) N, \\
\bar{\nabla}_{X} N & =-A_{N} X+\tau(X) N, \\
\nabla_{X} P Y & ={ }^{\star} \nabla_{X} P Y+C(X, P Y) \xi, \\
\nabla_{X} \xi & =-\stackrel{A}{A}_{\xi} X-\tau(X) \xi,
\end{aligned}
$$

for any $X, Y \in \Gamma(T M)$, where $\bar{\nabla}$ denotes the Levi-Civita connection on $(\bar{M}, \bar{g}), \nabla$ denotes the connection on $M$ induced from $\bar{\nabla}$ through the projection along the null transverse vector field $N$ and $\stackrel{\star}{\nabla}$ denotes the connection on the screen distribution $\mathscr{S}(\zeta)$ induced from $\nabla$ through the projection morphism $P$ of $\Gamma(T M)$ onto $\Gamma(\mathscr{S}(\zeta))$ with respect to the decomposition (2.4). Now the $(0,2)$ tensors $B$ and $C$ are the second fundamental forms on $T M$ and $\mathscr{S}(\zeta)$ respectively, $A_{N}$ and $\stackrel{\star}{\xi}_{\xi}$ are the shape operators on $T M$ with respect to the rigging $\zeta$ and the rigged vector field $\xi$ respectively and $\tau$ a 1-form on $T M$ defined by

$$
\tau(X)=\bar{g}\left(\bar{\nabla}_{X} N, \xi\right) .
$$


For the second fundamental forms $B$ and $C$ the following hold

$$
B(X, Y)=g\left(\stackrel{\star}{A}_{\xi} X, Y\right), \quad C(X, P Y)=g\left(A_{N} X, Y\right) \quad \forall X, Y \in \Gamma(T M),
$$

and

$$
B(X, \xi)=0, \quad \stackrel{\star}{A}_{\xi} \xi=0 \quad \text { and } \quad C(X, Y)-C(Y, X)=\langle[X, Y], N\rangle
$$

and the last equality shows that the screen structure $\mathscr{S}(\zeta)$ is integrable if and only if $C$ is symmetric on it. In this case, $\stackrel{\nabla}{\nabla}^{\star}$ is the Levi-Civita connection of the screen foliation from $(\bar{M}, \bar{g})$ and Equations (2.3) and (2.4) show that its second fundamental form is

$$
\mathbb{I}^{\zeta}(X, Y)=C(X, Y) \xi+B(X, Y) N, \quad X, Y \in \mathscr{S}(\zeta) .
$$

Let denote by $\bar{R}$ and $R$ the Riemannian curvature tensors of $\bar{\nabla}$ and $\nabla$, respectively. Then the following are the Gauss-Codazzi equations [20, p. 93].

$$
\begin{aligned}
\langle\bar{R}(X, Y) Z, \xi\rangle= & \left(\nabla_{X} B^{N}\right)(Y, Z)-\left(\nabla_{Y} B^{N}\right)(X, Z) \\
& +\tau^{N}(X) B^{N}(Y, Z)-\tau^{N}(Y) B^{N}(X, Z), \\
\langle\bar{R}(X, Y) Z, P W\rangle= & \langle R(X, Y) Z, P W\rangle+B^{N}(X, Z) C^{N}(Y, P W) \\
& -B^{N}(Y, Z) C^{N}(X, P W), \\
\langle\bar{R}(X, Y) \xi, N\rangle=\langle & \langle R(X, Y) \xi, N\rangle=C^{N}\left(Y, \hat{A}_{\xi} X\right)-C^{N}\left(X, \hat{A}_{\xi} Y\right) \\
\langle\bar{R}(X, Y) P Z, N\rangle= & -2 d \tau^{N}(X, Y), \quad \forall X, Y, Z, W \in \Gamma\left(\left.T M\right|_{\mathscr{U}}\right) . \\
& \left.\left.+\tau^{N}(Y)\left\langle A_{N}\right) Y, P Z\right\rangle-\left\langle\left(\nabla_{Y} A_{N}\right) X, P Z\right\rangle\right\rangle-\tau^{N}(X)\left\langle A_{N} Y, P Z\right\rangle .
\end{aligned}
$$

A null hypersurface $M$ is said to be totally umbilic (resp. totally geodesic) if there exists a smooth function $\rho$ on $M$ such that at each $p \in M$ and for all $u, v \in T_{p} M, B(p)(u, v)=\rho(p) \bar{g}(u, v)$ (resp. $B$ vanishes identically on $M$ ). These are intrinsic notions on any null hypersurface in the sense that they are independent of the normalization. Remark that $M$ is totally umbilic (resp. totally geodesic) if and only if $\stackrel{\star}{A}_{\xi}=\rho P$ (resp. $\stackrel{\star}{A}_{\xi}=0$ ). The trace of $\stackrel{\star}{A}_{\xi}$ is the null (non normalized) mean curvature of $M$, explicitly given by

$$
H_{p}=\sum_{i=2}^{n-1} \bar{g}\left(\stackrel{\star}{A}_{\xi}\left(e_{i}\right), e_{i}\right)=\sum_{i=2}^{n-1} B\left(e_{i}, e_{i}\right)
$$

being $\left(e_{2}, \ldots, e_{n-1}\right)$ an orthonormal basis of $\mathscr{S}(\zeta)$ at $p$. Let $\widetilde{\nabla}$ denote the Levi-Civita connection on the rigged Riemannian structure $(M, \widetilde{g})$. It holds [23],

$$
\left(L_{\xi} \widetilde{g}\right)(X, Y)=-2 B(X, Y), \quad X, Y \in \mathscr{S}(\zeta)
$$

In particular,

$$
H=-\widetilde{\operatorname{div}} \xi
$$

Observe that if $M$ is orientable compact without boundary it follows from (2.5) that $\int_{M} H d \bar{g}=0$.

Now, we recall from [18], the following generalized Raychaudhury equation,

$$
\overline{\operatorname{Ric}}(\xi)=\xi(H)+\tau(\xi) H-\left\|\hat{A}_{\xi}^{\star}\right\|^{2} .
$$

\section{Maximal null hypersurfaces in GRW spacetimes}

Due to the causal character $(0, n-2)$ of a null hypersurface in any $n$-dimensional $(n \geq 3)$ Lorentzian manifold, the normalization problem in a GRW spacetime has the outstanding feature that the Chen's closed timelike concircular vector field $\zeta=f \partial_{t}$ can act as rigging vector field for each of them. Let $\xi$ denote the corresponding rigged vector field. The associated null transverse vector field is

$$
N=f \partial_{t}+\frac{1}{2} f^{2}(t) \xi
$$


By the Weingarten formula, it holds

$$
-A_{N} X+\tau(X) N=f^{\prime}(t) X+\frac{1}{2}\left(X \cdot f^{2}\right) \xi+\frac{1}{2} f^{2}\left(-\stackrel{\star}{A}_{\xi} X-\tau(X) \xi\right) \quad \text { for } X \in \mathfrak{X}(M) .
$$

Hence, for $X \in \mathscr{S}(\zeta)$,

$$
\left(-A_{N} X+\frac{1}{2} f^{2} \stackrel{\star}{A}_{\xi} X-f^{\prime}(t) X\right)+\left(\frac{1}{2} f^{2} \tau(X)-(X \cdot f) f\right) \xi+\tau(X) N=0
$$

Then,

$$
\tau(X)=0, \quad X \cdot f=0 \quad \text { and } \quad A_{N} X=\frac{1}{2} f^{2} \stackrel{\star}{A}_{\xi} X-f^{\prime}(t) X
$$

for all $X \in \mathscr{S}(\zeta)$.

Now take $X=\xi$ in (3.1) and get

$$
A_{N} \xi=0, \quad \tau(\xi)=0 \quad \text { and } \quad \xi \cdot f=-\frac{f^{\prime}}{f}
$$

Then, we can state:

Proposition 3.1. Let $M$ be a null hypersurface in a GRW spacetime normalized with the Chen's vector field $\zeta=f \partial_{t}$. Then,

(i) the 1-form $\tau$ vanishes identically on $M$ and the rigged vector field $\xi$ is geodesic.

(ii) $\xi \cdot f=-\frac{f^{\prime}}{f}$ and for all vector field $X$ tangent to the screen structure $\mathscr{S}(\zeta)$, it holds $X \cdot f=0$.

(iii) For all $X \in \mathfrak{X}(M)$,

$$
A_{N} X=\frac{1}{2} f^{2} \stackrel{\star}{A}_{\xi} X^{\mathscr{S}}-f^{\prime}(t) X^{\mathscr{S}}
$$

where $X^{\mathscr{S}}=P X$ with $P$ the projection morphism of $\Gamma(T M)$ onto $\Gamma(\mathscr{S}(\zeta))$ with respect to the decomposition (2.4).

Since $\zeta$ is closed, the screen structure $\mathscr{S}(\zeta)$ induces a foliation on $M$. For $p=(t, x) \in M$ let $\mathscr{F}_{p}$ denote the leaf of $\mathscr{S}(\zeta)$ through p. Every $X \in \mathfrak{X}(M)$ splits as follows

$$
X=-\frac{1}{f} \alpha(X) \partial_{t}+X^{F}
$$

where $X^{F}$ is the lift of the projection of $X$ onto the fiber $F$ and $\alpha=\bar{g}(\zeta, \cdot)=-f d t$ is the 1 -form metrically equivalent to $\zeta$ respect to $\bar{g}$. It follows that $X \in \mathfrak{X}(M)$ is tangent to the screen structure if and only if $X=X^{F} \in \mathfrak{X}(F)$. In other words, each leaf $\mathscr{F}_{p}$ is a hypersurface in the slice $\left\{\pi_{I}(p)\right\} \times F$. Furthermore, for $X \in \mathfrak{X}(M)$, it holds $X=\alpha(X) \xi+X^{\mathscr{S}}$ which gives

$$
X^{F}=X^{\mathscr{S}}+\alpha(X) \xi^{F}
$$

where $\xi=-\frac{1}{f} \partial_{t}+\xi^{F}$. In particular $g_{F}\left(X^{\mathscr{S}}, \xi^{F}\right)=0$. Then, since $g_{F}\left(\xi^{F}, \xi^{F}\right)=\frac{1}{f^{4}}$ it follows that at each point $p=(t, x) \in M$, the vector $f(t) \xi^{F}$ is a unit normal in $\{t\} \times F$ to the leaf $\mathscr{F}_{p}$ of $\mathscr{S}(\zeta)$ through $p$. Let $A_{f \xi^{F}}^{F}$ and $H^{\mathscr{F}}$ denote respectively the shape operator and the mean curvature of $\mathscr{F}_{p}$ as a hypersurface of the slice $\{t\} \times F$ which inherits the metric $f^{2}(t) g_{F}$.

Theorem 3.2. Let $I \times_{f} F$ be a GRW spacetime. A null hypersurface $M$ is maximal if and only if the screen foliation induced by the Chen's vector field $\zeta=f \partial_{t}$ has constant mean curvature $-\frac{f^{\prime}(t)}{f(t)}$ in each slice $\{t\} \times F$.

Proof. Given $X \in \mathfrak{X}(M)$, we have

$$
\begin{aligned}
-\stackrel{\star}{A}_{\xi} X & =\bar{\nabla}_{X} \xi=\bar{\nabla}_{X}\left(-\frac{1}{f} \partial_{t}+\xi^{F}\right)=\frac{X \cdot f}{f^{2}} \partial_{t}-\frac{1}{f} \bar{\nabla}_{X} \partial_{t}+\bar{\nabla}_{X} \xi^{F} \\
& =\frac{X \cdot f}{f^{2}} \partial_{t}-\frac{1}{f^{2}}\left[\bar{\nabla}_{X} f \partial_{t}-(X \cdot f) \partial_{t}\right]+\bar{\nabla}_{X} \xi^{F} \\
& =2 \frac{X \cdot f}{f^{2}} \partial_{t}-\frac{f^{\prime}}{f^{2}} X-\frac{1}{f} \alpha(X) \bar{\nabla}_{\partial_{t}} \xi^{F}+\bar{\nabla}_{X^{F}} \xi^{F} \\
& =2 \frac{X \cdot f}{f^{2}} \partial_{t}-\frac{f^{\prime}}{f^{2}} X-\frac{f^{\prime}}{f} \alpha(X) \xi^{F}+\bar{\nabla}_{X^{F}} \xi^{F}
\end{aligned}
$$

As $\stackrel{\star}{A}_{\xi} \xi=0$, we just need to compute (3.3) for $X \in \mathscr{S}(\zeta)$ i.e $X=X^{F}=X^{\mathscr{S}}$ for which $X \cdot f=0$ as seen from item (ii) in Proposition 3.1 and $\alpha(X)=0$. Let $\nabla^{F}$ denote the Levi-Civita connection of $\left(F, g_{F}\right)$. We have for all $X \in \mathscr{S}(\zeta)$,

$$
\stackrel{\star}{A}_{\xi} X=\frac{f^{\prime}}{f^{2}} X-\bar{\nabla}_{X} \xi^{F}=\frac{f^{\prime}}{f^{2}} X-\frac{1}{f} \bar{\nabla}_{X} f \xi^{F}
$$




$$
=\frac{f^{\prime}}{f^{2}} X-\frac{1}{f}\left[\nabla_{X}^{F} f \xi^{F}+\left\langle X, f \xi^{F}\right\rangle \frac{f^{\prime}}{f} \partial_{t}\right],
$$

thus for all $X \in \mathscr{S}(\zeta)$,

$$
\stackrel{\star}{A_{\xi}} X=\frac{f^{\prime}}{f^{2}} X+\frac{1}{f} A_{f \xi}^{F} X .
$$

Let $\left(\partial_{t}, f \xi^{F}, e_{2}, \ldots, e_{n-1}\right)$ be a frame field on $\bar{M}$ along $M$ such that $\left(e_{2}, \ldots, e_{n-1}\right)$ represents an orthonormal basis for $\mathscr{S}(\zeta)$. The null mean curvature $H_{p}$ at $p=(t, x)$ is given by

$$
\begin{aligned}
H_{p} & =\sum_{i=2}^{n-1}\left\langle A_{\xi}^{\star} e_{i}, e_{i}\right\rangle=\sum_{i=2}^{n-1}\left[\frac{f^{\prime}}{f^{2}}\left\langle e_{i}, e_{i}\right\rangle+\frac{1}{f}\left\langle A_{f \xi^{F}}^{F} e_{i}, e_{i}\right\rangle\right] \\
& =(n-2) \frac{f^{\prime}}{f^{2}}+\frac{n-2}{f} H^{\mathscr{F}}
\end{aligned}
$$

that is

$$
H_{p}=\frac{n-2}{f^{2}}\left[f^{\prime}(t)+f(t) H^{\mathscr{F}}\right]
$$

and the claim follows from (3.5).

In the way of above proof, we have established (combining (3.4) and $\stackrel{\star}{A_{\xi}} \xi=0$ ) the following:

Proposition 3.3. Let $I \times_{f} F$ be a GRW spacetime. A null hypersurface $M$ is totally umbilic if and only if the screen foliation induced by the Chen's vector field $\zeta=f \partial_{t}$ is totally umbilic in the slices.

Theorem 3.4. Let $I \times_{f} F$ be a GRW spacetime and suppose $M$ is a maximal null hypersurface normalized by the Chen's vector field $\zeta=f \partial_{t}$.

(i) The squared norm of the screen shape operator has the following upper bound

$$
\left\|\stackrel{\star}{A}_{\xi}\right\|^{2} \leq \frac{n-2}{f^{2}} W(\zeta, \xi, \zeta, \xi)+\frac{1}{(n-1) f^{4}}\left[(n-1)(n-2) f f^{\prime \prime}-s_{F}\right]
$$

with equality if and only if the scale factor $f$ is constant, in particular the slices are minimal.

(ii) If the warping function $f$ has a convex logarithm i.e $(\ln f)^{\prime \prime} \geq 0\left(\right.$ resp. a concave logarithm, i.e $\left.(\ln f)^{\prime \prime} \leq 0\right)$ then

$$
\begin{gathered}
\left\|\star_{\xi}^{\star}\right\|^{2} \geq \frac{n-2}{f^{2}} W(\zeta, \xi, \zeta, \xi)-\frac{1}{(n-1) f^{4}} s_{F} \\
\left(\text { resp. }\left\|\stackrel{\star}{A}_{\xi}\right\|^{2} \leq \frac{n-2}{f^{2}} W(\zeta, \xi, \zeta, \xi)-\frac{1}{(n-1) f^{4}} s_{F}\right)
\end{gathered}
$$

with equality if and only if the warping function is given by

$$
f(t)=k \exp (\lambda t), \quad k \in \mathbb{R}_{+}^{\star}, \quad \lambda \in \mathbb{R} .
$$

(iii) If the Weyl tensor satisfies $i_{\partial_{t}} W=0$, then the Null Convergence Condition (NCC) holds if and only if the scalar curvature $s_{F}$ of the fiber $F$ has the following lower bound

$$
s_{F} \geq(n-1)(n-2) f^{2}(\ln f)^{\prime \prime},
$$

in which case the GRW spacetime admits no non totally geodesic maximal null hypersurface. Otherwise, on the set of non geodesic points it holds

$$
\left\|\stackrel{\star}{A}_{\xi}\right\|^{2} \leq \frac{1}{(n-1) f^{4}}\left[(n-1)(n-2) f f^{\prime \prime}-s_{F}\right]
$$

with

$$
s_{F}<(n-1)(n-2) f f^{\prime \prime} .
$$


Proof. From (2.1) and (2.2) it is easy to see that the eigenvalue $\sigma$ associated to the Chen's vector $\zeta$ and the scalar curvature $\bar{s}$ are given by

$$
\sigma=(n-1) \frac{f^{\prime \prime}}{f}
$$

and

$$
\bar{s}=2(n-1) \frac{f^{\prime \prime}}{f}+(n-1)(n-2) \frac{f^{\prime 2}}{f^{2}}+\frac{s_{F}}{f^{2}} .
$$

It follows from (2.1) that for all null vector $U$,

$$
\overline{\operatorname{Ric}}(U)=-(n-2) W\left(\partial_{t}, U, \partial_{t}, U\right)-\frac{\left\langle\partial_{t}, U\right\rangle^{2}}{n-1}\left[(n-1)(n-2)(\ln f)^{\prime \prime}-\frac{s_{F}}{f^{2}}\right] .
$$

In particular,

$$
\overline{\operatorname{Ric}}(\xi)=-\frac{(n-2)}{f^{2}} W(\zeta, \xi, \zeta, \xi)-\frac{1}{(n-1) f^{4}}\left[(n-1)(n-2) f^{2}(\ln f)^{\prime \prime}-s_{F}\right]
$$

It follows from (3.10) and (2.6) that for a maximal $M$,

$$
\left\|\stackrel{\star}{A}_{\xi}\right\|^{2}=-\overline{\operatorname{Ric}}(\xi)=\frac{n-2}{f^{2}} W(\zeta, \xi, \zeta, \xi)+\frac{1}{(n-1) f^{4}}\left[(n-1)(n-2) f^{2}(\ln f)^{\prime \prime}-s_{F}\right]
$$

which gives (3.6) with equality if and only if $f^{\prime}=0$ in particular the slices (with mean curvature $\frac{f^{\prime}(t)}{f(t)}$ ) are minimal and (i) is proved. For (ii), since $n \geq 3$, the hypothesis implies $(n-1)(n-2) f^{2}(\ln f)^{\prime \prime} \geq 0\left(\right.$ resp. $\left.(n-1)(n-2) f^{2}(\ln f)^{\prime \prime} \leq 0\right)$. The equality case for both estimations is obtained for $(\ln f)^{\prime \prime}=0$ that is $f(t)=k \exp (\lambda t), \quad k \in \mathbb{R}_{+}^{\star}, \quad \lambda \in \mathbb{R}$.

Suppose the Weyl tensor satisfies $i_{\partial_{t}} W=0$. Then, from (3.9) the null convergence condition holds if and only if the scalar curvature $s_{F}$ of the fiber satisfies (3.7). In this case, using (2.6) for a maximal $M$ leads to $-\|\stackrel{\star}{A} \xi\|^{2}=\overline{\operatorname{Ric}}(\xi) \geq 0$ i.e $\stackrel{\star}{A} \xi_{\xi}=0$ and $M$ is totally geodesic. Otherwise (i.e if the null convergence condition failed), for the set of non geodesic points,

$$
s_{F}<(n-1)(n-2) f^{2}(\ln f)^{\prime \prime}=(n-1)(n-2)\left(f f^{\prime \prime}-f^{\prime 2}\right) \leq(n-1)(n-2) f f^{\prime \prime}
$$

and (3.8) follows from (3.6) in which the first term vanishes.

\section{A generic example}

Let $I \times_{f} F$ be a GRW spacetime and consider $\psi: F \longrightarrow I$ a differentiable function with graph

$$
M=\{(\psi(x), x), \quad x \in F\} .
$$

This is a null hypersurface if and only if

$$
\left\|\operatorname{grad}^{F} \psi\right\|_{F}=f \circ \psi
$$

that is $\psi$ is a generalized eikonal function. In this case, a rigging for it is

$$
\zeta=(f \circ \psi) \partial_{t}
$$

with associated rigged vector field given by

$$
\xi=-\frac{1}{f \circ \psi} \partial_{t}-\frac{1}{(f \circ \psi)^{3}} \operatorname{grad}^{F} \psi .
$$

In particular $\xi^{F}=-\frac{1}{(f \circ \psi)^{3}} \operatorname{grad}^{F} \psi$. The screen structure is given by the level sets of $\psi$ : for each $p=\left(t_{0}, x_{0}\right) \in M, \mathscr{F}_{p}=$ $\left\{t_{0}\right\} \times \psi^{-1}\left(t_{0}\right)$. Let us compute the second fundamental form and screen shape operator for $\left\{t_{0}\right\} \times_{f} \psi^{-1}\left(t_{0}\right)$ relative to $\left\{t_{0}\right\} \times{ }_{f} F$ Let

$$
u_{\psi}=-\frac{1}{(f \circ \psi)^{2}} \operatorname{grad}^{F} \psi
$$


As per (4.1), $u_{\psi}$ is a unit normal vector to $\mathscr{F}_{(t, x)}$ in $\{t\} \times_{f} F$ and we have

$$
\xi=-\frac{1}{f \circ \psi} \partial_{t}+\frac{1}{(f \circ \psi)} u_{\psi}=\frac{1}{(f \circ \psi)}\left[-\partial_{t}+u_{\psi}\right] .
$$

Then, for any $X \in \mathfrak{X}(M)$,

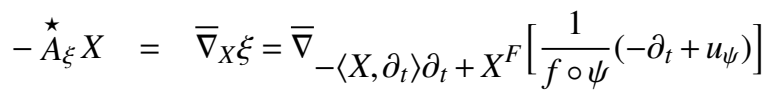

$$
\begin{aligned}
& =-\left\langle X, \partial_{t}\right\rangle \frac{1}{f \circ \psi} \frac{f^{\prime} \circ \psi}{f \circ \psi} u_{\psi}-\frac{1}{f \circ \psi} \frac{f^{\prime} \circ \psi}{f \circ \psi} X^{F}+\frac{X^{F} \cdot(f \circ \psi)}{(f \circ \psi)^{2}} \partial_{t} \\
& +\frac{1}{f \circ \psi} \nabla_{X^{F}}^{F} u_{\psi}+\left(f^{\prime} \circ \psi\right) g_{F}\left(X^{F}, u_{\psi}\right) \partial_{t}-\frac{X^{F} \cdot(f \circ \psi)}{(f \circ \psi)^{2}} u_{\psi} \text {. }
\end{aligned}
$$

But the left hand side belongs to the screen structure, which is orthogonal to $\zeta=f \partial_{t}$, so it holds

$$
\frac{X^{F} \cdot(f \circ \psi)}{(f \circ \psi)^{2}}=-\left(f^{\prime} \circ \psi\right) g_{F}\left(X^{F}, u_{\psi}\right)=-\frac{f^{\prime} \circ \psi}{(f \circ \psi)^{2}}\left\langle\partial_{t}, X\right\rangle,
$$

that is

$$
X^{F} \cdot(f \circ \psi)=-\left(f^{\prime} \circ \psi\right)\left\langle\partial_{t}, X\right\rangle
$$

where in (4.3) we use $\left\langle\partial_{t}, X\right\rangle=\left\langle X^{F}, u_{\psi}\right\rangle=(f \circ \psi)^{2} g_{F}\left(X^{F}, u_{\psi}\right)$ due to $\langle X, \xi\rangle=0$.

Then,

$$
\begin{aligned}
\stackrel{\star}{A}_{\xi} X & =\left\langle X, \partial_{t}\right\rangle \frac{1}{f \circ \psi} \frac{f^{\prime} \circ \psi}{f \circ \psi} u_{\psi}+\frac{1}{f \circ \psi} \frac{f^{\prime} \circ \psi}{f \circ \psi} X^{F}-\frac{1}{f \circ \psi} \nabla_{X^{F}}^{F} u_{\psi}+\frac{X^{F} \cdot(f \circ \psi)}{(f \circ \psi)^{2}} u_{\psi} \\
\stackrel{(4.3)}{=} & \frac{f^{\prime} \circ \psi}{(f \circ \psi)^{2}} X^{F}-\frac{1}{f \circ \psi} \nabla_{X^{F}}^{F} u_{\psi} .
\end{aligned}
$$

Replacing $u_{\psi}$ leads to

$$
\stackrel{\star}{A}_{\xi} X=\frac{1}{(f \circ \psi)^{3}} \nabla_{X^{F}}^{F} \operatorname{grad}^{F} \psi+\frac{f^{\prime} \circ \psi}{(f \circ \psi)^{2}} X^{F}+2 \frac{f^{\prime} \circ \psi}{(f \circ \psi)^{4}}\left\langle X, \partial_{t}\right\rangle \operatorname{grad}^{F} \psi
$$

In particular, for any $X \in \mathscr{S}(\zeta)$,

$$
\stackrel{\star}{A}_{\xi} X=\frac{1}{(f \circ \psi)^{3}} \nabla_{X}^{F} \operatorname{grad}^{F} \psi+\frac{f^{\prime} \circ \psi}{(f \circ \psi)^{2}} X .
$$

Hence, for $X, Y \in \mathfrak{X}(M)$,

$$
\begin{aligned}
B(X, Y)= & \frac{1}{f \circ \psi} \operatorname{Hess}_{\psi}^{F}\left(X^{F}, Y^{F}\right)+\left(f^{\prime} \circ \psi\right) g_{F}\left(X^{F}, Y^{F}\right) \\
& +2\left\langle X, \partial_{t}\right\rangle \frac{f^{\prime} \circ \psi}{(f \circ \psi)^{2}} d \psi\left(Y^{F}\right)
\end{aligned}
$$

Then the restriction $B_{\mid \mathscr{S}(\zeta) \times \mathscr{S}(\zeta)}$ of the second fundamental form reads for all $X, Y \in \mathscr{S}(\zeta)$,

$$
B(X, Y)=\frac{1}{f \circ \psi} \operatorname{Hess}_{\psi}^{F}(X, Y)+\left(f^{\prime} \circ \psi\right) g_{F}(X, Y)
$$

Consider a quasi $\bar{g}$-orthonormal frame field $\left(\partial_{t}, f \xi^{F}, e_{2}, \ldots, e_{n-1}\right)$ on $\bar{M}$ with $\left(e_{i}\right)_{2 \leq i \leq n-1}$ tangent to $\mathscr{F}$. Then, $\left(f^{2} \xi^{F}, f e_{2}, \ldots, f e_{n-1}\right)$ is an orthonormal frame field for $g_{F}$. Therefore, the null mean curvature reads

$$
\begin{aligned}
H & =\sum_{i=2}^{n-1} B\left(e_{i}, e_{i}\right) \\
& =\frac{1}{f \circ \psi} \sum_{i=2}^{n-1} g_{F}\left(\nabla_{e_{i}}^{F} \operatorname{grad}^{F} \psi, e_{i}\right)+\left(f^{\prime} \circ \psi\right) \sum_{i=2}^{n-1} g_{F}\left(e_{i}, e_{i}\right)
\end{aligned}
$$




$$
\begin{aligned}
= & \frac{1}{(f \circ \psi)^{3}} \sum_{i=2}^{n-1} g_{F}\left(\nabla_{f e_{i}}^{F} \operatorname{grad}^{F} \psi, f e_{i}\right)+\frac{f^{\prime} \circ \psi}{(f \circ \psi)^{2}} \sum_{i=2}^{n-1} g_{F}\left(f e_{i}, f e_{i}\right) \\
= & \frac{1}{(f \circ \psi)^{3}}\left[\sum_{i=2}^{n-1} g_{F}\left(\nabla_{f e_{i}}^{F} \operatorname{grad}^{F} \psi, f e_{i}\right)+g_{F}\left(\nabla_{f^{2} \xi^{F}}^{F} \operatorname{grad}^{F} \psi, f^{2} \xi^{F}\right)\right] \\
& -\frac{1}{(f \circ \psi)^{3}} g_{F}\left(\nabla_{f^{2} \xi^{F}}^{F} \operatorname{grad}^{F} \psi, f^{2} \xi^{F}\right)+(n-2) \frac{f^{\prime} \circ \psi}{(f \circ \psi)^{2}} \\
= & \frac{1}{(f \circ \psi)^{3}} \Delta^{F} \psi+(n-2) \frac{f^{\prime} \circ \psi}{(f \circ \psi)^{2}} \\
& -\frac{1}{(f \circ \psi)^{3}} g_{F}\left(\nabla_{f^{2} \xi^{F}}^{F} \operatorname{grad}^{F} \psi, f^{2} \xi^{F}\right) .
\end{aligned}
$$

Let us compute the term $g_{F}\left(\nabla_{(f \circ \psi)^{2} \xi^{F}}^{F} \operatorname{grad}^{F} \psi,(f \circ \psi)^{2} \xi^{F}\right)$. Note that from (4.2) $(f \circ \psi)^{2} \xi^{F}=-\frac{1}{f \circ \psi} \operatorname{grad}^{F} \psi$. So,

$$
\begin{aligned}
g_{F}\left(\nabla_{(f \circ \psi)^{2} \xi^{F}}^{F} \operatorname{grad}^{F} \psi,(f \circ \psi)^{2} \xi^{F}\right) & =-(f \circ \psi) g_{F}\left(\nabla_{\xi^{F}}^{F} \operatorname{grad}^{F} \psi, \operatorname{grad}^{F} \psi\right) \\
& =-\frac{(f \circ \psi)}{2} \xi^{F} \cdot\left(\left\|\operatorname{grad}^{F} \psi\right\|_{F}^{2}\right) \\
& =-(f \circ \psi)^{2} \xi^{F} \cdot(f \circ \psi) \\
\stackrel{(4.3)}{=} & (f \circ \psi)^{2} \frac{f^{\prime} \circ \psi}{f \circ \psi}=\left(f^{\prime} \circ \psi\right)(f \circ \psi) .
\end{aligned}
$$

It follows that

$$
H=\frac{1}{(f \circ \psi)^{3}}\left[\Delta^{F} \psi+(n-3)\left(f^{\prime} \circ \psi\right)(f \circ \psi)\right] .
$$

From (4.4) we can state the following:

Theorem 4.1. Let $I \times_{f} F$ be a GRW spacetime and consider $\psi: F \longrightarrow I$ a differentiable function with graph

$$
M=\{(\psi(x), x), \quad x \in F\} \quad \text { such that }\left\|\operatorname{grad}^{F} \psi\right\|_{F}=f \circ \psi
$$

(i) $M$ is a null hypersurface which is maximal if and only if

$$
\Delta^{F} \psi=-(n-3)\left(f^{\prime} \circ \psi\right)(f \circ \psi)
$$

(ii) If $M$ is maximal then $\Delta^{F} \psi$ is constant on each leaf $\mathscr{F}_{p}, p \in M$.

(iii) The (Riemannian) mean curvature $H^{\mathscr{F}}$ of a leaf $\mathscr{F}_{p}$ as a hypersurface in the slice $\left\{\pi_{I}(p)\right\} \times F$ is given by

$$
H^{\mathscr{F}}=\frac{1}{(n-2)(f \circ \psi)^{2}}\left[\Delta^{F} \psi-\left(f^{\prime} \circ \psi\right)(f \circ \psi)\right] .
$$

It follows that leaves of the foliation $\mathscr{F}$ has constant mean curvature if and only if $\Delta^{F} \psi$ is constant leafwise. In particular they are minimal if and only if $\Delta^{F} \psi_{\left.\right|_{\mathscr{F}}}=\left(f^{\prime} \circ \psi\right)(f \circ \psi)$.

Proof. The first claim (i) follows from (4.1) and (4.4). For (ii) just recall that $\psi$ is constant on each leaf of the foliation $\mathscr{F}$. Now, for (iii), use (3.5) and (4.4) to derive (4.5).

\section{The extrinsic scalar curvature estimates}

In [19] we pointed out that there are no natural ways to induce a scalar curvature analogue on null hypersurfaces (or more generally a null submanifolds) as usual. The drawback in considering this concept on null hypersurfaces is twofold: since the induced connection is not a Levi-Civita connection (unless $M$ be totally geodesic) the $(0,2)$ induced Ricci tensor is not symmetric in general. Also, as the induced metric is degenerate, its inverse does not exist and it is not possible to proceed in the usual way by contracting the Ricci tensor to get a scalar quantity. A first attempt in this way was made in [27] which consists to restrict the concept to a very limited class of null hypersurfaces: those admitting a "canonical screen distribution" that induces a canonical transversal vector bundle and a symmetric induced Ricci tensor. Although the above two conditions are interesting to compensate lacking due to the above quoted difficulties, to admit symmetric induced Ricci tensor in lightlike setting is very restrictive. Also, the problem in contracting with respect to the noninvertible induced metric is still unsolved for the general setting. However, when the null hypersurface is provided with a normalization (a rigging), thanks to the nondegenerate associated rigged structure, we can drop above restrictions and construct an analog of this scalar quantity (see [19]) called extrinsic scalar curvature, referring to the use of an extra structure $\zeta$, the rigging. 
Our purpose in this section is to determine this scalar quantity for null hypersurfaces in GRW spacetimes, normalized by the Chen's vector field $\zeta=f \partial_{t}$ and give some bounds for it in case the null hypersurface is maximal.

Let Ric denote the induced Ricci tensor on the normalized $(M, \zeta)$. We define the symmetrized $(0,2)-$ Ricci tensor Ric $^{\text {sym }}$ by

$$
\operatorname{Ric}^{\operatorname{sym}}(X, Y)=\frac{1}{2}[\operatorname{Ric}(X, Y)+\operatorname{Ric}(Y, X)] \text {. }
$$

By direct computation ([19]), we see that

$$
\begin{aligned}
\operatorname{Ric}^{\text {sym }}(X, Y)= & \overline{\operatorname{Ric}}(X, Y)+B(X, Y) \operatorname{tr}\left(A_{N}\right) \\
& -\frac{1}{2}\left[\langle\bar{R}(\xi, X) Y, N\rangle+\langle\bar{R}(\xi, Y) X, N\rangle+\left\langle A_{N} X, \stackrel{\star}{A}_{\xi} Y\right\rangle+\left\langle A_{N} Y, \stackrel{\star}{A}_{\xi} X\right\rangle\right]
\end{aligned}
$$

Now, the extrinsic scalar curvature $s_{\zeta}$ on $(M, \zeta)$ is the $\widetilde{g}$-trace of $R i c^{\text {sym }}$, where $\widetilde{g}$ is the associated Riemannian rigged structure, i.e

$$
s_{\zeta}=\widetilde{g}^{\alpha \beta} \operatorname{Ric}_{\alpha \beta}^{s y m}
$$

being $\left(e_{1}=\xi, e_{2}, \ldots, e_{n-1}\right)$ a $\widetilde{g}-$ orthonormal frame field on $M$ with $\left(e_{i}\right)_{1 \leq i \leq n-1}$ tangent to the screen structure. We compute the components $\operatorname{Ric}_{\alpha \beta}^{\text {sym }}$ using (5.1), (3.2) and symmetries in (2.2).

$$
\begin{aligned}
& \operatorname{Ric}_{00}^{s y m}=\operatorname{Ric}^{\text {sym }}(\xi, \xi)=\overline{\operatorname{Ric}}(\xi, \xi)=\overline{\operatorname{Ric}}\left(-\frac{1}{f} \partial_{t}+\xi^{F},-\frac{1}{f} \partial_{t}+\xi^{F}\right) \\
& =-(n-1) \frac{f^{\prime \prime}}{f^{3}}+\frac{f f^{\prime \prime}+(n-2) f^{\prime 2}}{f^{4}}+\operatorname{Ric}^{F}\left(\xi^{F}, \xi^{F}\right) \\
& =\frac{2-n}{f^{2}}(\ln f)^{\prime \prime}+\operatorname{Ric}^{F}\left(\xi^{F}, \xi^{F}\right) \text {. } \\
& \operatorname{Ric}_{0 i}^{\text {sym }}=\operatorname{Ric}^{\text {sym }}\left(\xi, e_{i}\right)=\overline{\operatorname{Ric}}\left(\xi, e_{i}\right)-\frac{1}{2}\left\langle\bar{R}\left(\xi, e_{i}\right) \xi, N\right\rangle \\
& =\operatorname{Ric}^{F}\left(\xi^{F}, e_{i}\right) \\
& \operatorname{Ric}_{i j}^{s y m}=\operatorname{Ric}^{\text {sym }}\left(e_{i}, e_{j}\right)=\overline{\operatorname{Ric}}\left(e_{i}, e_{j}\right)+B\left(e_{i}, e_{j}\right) \operatorname{tr}\left(A_{N}\right) \\
& -\frac{1}{2}\left(\left\langle f \partial_{t}+\frac{1}{2} f^{2} \xi, \bar{R}\left(\xi, e_{j}\right) e_{i}\right\rangle\right. \\
& \left.+\left\langle f \partial_{t}+\frac{1}{2} f^{2} \xi, \bar{R}\left(\xi, e_{i}\right) e_{j}\right\rangle\right) \\
& +\left\langle A_{N} e_{i}, \stackrel{\star}{A}_{\xi} e_{j}\right\rangle++\left\langle A_{N} e_{j}, \stackrel{\star}{A}_{\xi} e_{i}\right\rangle
\end{aligned}
$$

Then, using the following relations of curvature tensor [28],

$$
\begin{aligned}
\bar{R}\left(\partial_{t}, \partial_{t}\right) \partial_{t} & =\bar{R}\left(\partial_{t}, \partial_{t}\right) X^{F}=\bar{R}\left(X^{F}, Y^{F}\right) \partial_{t}=0 \\
\bar{R}\left(X^{F}, \partial_{t}\right) \partial_{t} & =-\frac{f^{\prime \prime}}{f} X^{F}, \quad \bar{R}\left(\partial_{t}, X^{F}\right) Y^{F}=f f^{\prime \prime} g_{F}\left(X^{F}, Y^{F}\right) \partial_{t} \\
\bar{R}\left(X^{F}, Y^{F}\right) Z^{F} & =R^{F}\left(\left(X^{F}, Y^{F}\right) Z^{F}\right)+f^{\prime 2}\left[g_{F}\left(X^{F}, Z^{F}\right) Y^{F}-g_{F}\left(Y^{F}, Z^{F}\right) X^{F}\right]
\end{aligned}
$$

we have

$$
\begin{aligned}
\operatorname{Ric}^{s y m}\left(e_{i}, e_{j}\right)= & \overline{\operatorname{Ric}}\left(e_{i}, e_{j}\right)+B\left(e_{i}, e_{j}\right) \operatorname{tr}\left(A_{N}\right) \\
& -\frac{1}{2}\left(2 f f^{\prime \prime} g_{F}\left(e_{i}, e_{j}\right)+\frac{1}{2}\left\langle R^{F}\left(\xi^{F}, e_{j}\right) e_{i}, \xi^{F}\right\rangle\right. \\
& +\frac{1}{2}\left\langle R^{F}\left(\xi^{F}, e_{i}\right) e_{j}, \xi^{F}\right\rangle-2 \frac{f^{\prime 2}}{f^{2}} g_{F}\left(e_{i}, e_{j}\right) \\
& \left.+\left\langle\stackrel{\star}{A}_{\xi} A_{N} e_{i}, e_{j}\right\rangle+\left\langle\stackrel{\star}{A}_{\xi} A_{N} e_{j}, e_{i}\right\rangle\right)
\end{aligned}
$$


Now, relation (iii) in Proposition 3.1 implies

$$
\left\langle\stackrel{\star}{A}_{\xi} A_{N} e_{j}, e_{i}\right\rangle=\frac{1}{2} f^{2}\left\langle\stackrel{\star}{A}_{\xi}^{2} e_{i}, e_{j}\right\rangle-f^{\prime} B\left(e_{i}, e_{j}\right)
$$

and

$$
\operatorname{tr}\left(A_{N}\right)=\frac{1}{2} f^{2} H-(n-2) f^{\prime}
$$

Then,

$$
\begin{aligned}
\operatorname{Ric}^{\text {sym }}\left(e_{i}, e_{j}\right)= & \operatorname{Ric}^{F}\left(e_{i}, e_{j}\right)+\left[(n-2)+\frac{1}{f^{2}}\right] f^{\prime 2} g_{F}\left(e_{i}, e_{j}\right) \\
& +\left[\frac{1}{2} f^{2} H+(3-n) f^{\prime}\right] B\left(e_{i}, e_{j}\right) \\
& -\frac{1}{2} f^{2}\left[\left\langle R^{F}\left(e_{i}, \xi^{F}\right) \xi^{F}, e_{j}\right\rangle+\left\langle\stackrel{\star}{A_{\xi}} e_{i}, \stackrel{\star}{A_{\xi}} e_{j}\right\rangle\right]
\end{aligned}
$$

Also, the components $\widetilde{g}^{a b}$ in the same frame field are the following:

$$
\widetilde{g}^{00}=1, \quad \widetilde{g}^{0 i}=\widetilde{g}^{i 0}=0, \quad \widetilde{g}^{i j}=\frac{1}{f^{2}} g_{F}^{i j} .
$$

Finally, after substitution in (5.2) and reducing we get the following expression for the extrinsic scalar curvature $s_{\zeta}$ on the normalized $(M, \zeta)$.

Proposition 5.1. Let $I \times_{f} F$ be a GRW spacetime and suppose $M$ is a null hypersurface normalized by the Chen's vector field $\zeta=f \partial_{t}$. Then the extrinsic scalar curvature on $(M, \zeta)$ is given by

$$
\begin{aligned}
s_{\zeta}= & \frac{1}{f^{4}} s_{F}+\left[\frac{1}{2} f^{2} H+(3-n) f^{\prime}\right] H \\
& -\frac{1}{2}\left[\operatorname{Ric}^{F}\left(\xi^{F}, \xi^{F}\right)+f^{2}\|\stackrel{\star}{A} \xi\|^{2}\right] \\
& +\frac{2-n}{f^{2}}(\ln f)^{\prime \prime}+\frac{n-2}{f^{2}}[(n-2) f+1](\ln f)^{\prime}
\end{aligned}
$$

Theorem 5.2. Let $I \times_{f} F$ be a $n$-dimensional GRW spacetime $(n \geq 3)$ and $M$ a maximal null hypersurface normalized with the Chen's vector field $\zeta=f \partial_{t}$.

(i) If $f$ is a convex decreasing warping function then $s_{\zeta}$ has the following upper bound

$$
s_{\zeta} \leq \frac{1}{f^{4}} s_{F}-\frac{1}{2} \operatorname{Ric}^{F}\left(\xi^{F}, \xi^{F}\right) .
$$

(ii) If $i_{\zeta} W=0$ (a quasi-Einstein space), then on the set of non totally geodesic points of a maximal null hypersurface, it holds

$$
\begin{aligned}
s_{\zeta} \geq & \frac{1}{f^{4}}\left[1+\frac{f^{2}}{2(n-1)}\right] s_{F}+-\frac{1}{2} \operatorname{Ric}^{F}\left(\xi^{F}, \xi^{F}\right) \\
& -\frac{n-2}{f^{4}}\left[\frac{1}{2} f^{3} f^{\prime \prime}+f f^{\prime \prime}-f^{\prime 2}-((n-2) f+1) f f^{\prime}\right] .
\end{aligned}
$$

Proof. Item (i) is immediate using (5.3). Indeed, we have $H=0$ (maximality), $-\frac{1}{2} f^{2}\left\|\stackrel{\star}{\xi}_{\xi}\right\|^{2}<0$ and as $n \geq 3$ the hypothesis on the warping function $f$ leads to $\frac{2-n}{f^{2}}(\ln f)^{\prime \prime}+\frac{n-2}{f^{2}}[(n-2) f+1](\ln f)^{\prime}<0$. For (ii), the hypothesis implies from (3.8) that

$$
-\frac{1}{2} f^{2}\left\|\stackrel{\star}{A}_{\xi}\right\|^{2} \geq \frac{-1}{2(n-1) f^{2}}\left[(n-1)(n-2) f f^{\prime \prime}-s_{F}\right] .
$$

Then relation (5.4) follows from (5.3). 


\section{Willmore null hypersurfaces}

\subsection{The Willmore functional}

For a normalized null hypersurface $(M, \zeta)$ with compactly supported (non normalized) mean curvature $H$ (in particular if $M$ is compact), the Willmore action is given by

$$
\mathcal{W}(M, \zeta)=\int_{M} H^{2} d M
$$

where $d M$ is the volume element induced on $M$.

An obvious fact is that

$$
H^{2} \leq(n-2)\left\|\stackrel{\star}{A}_{\xi}\right\|^{2}
$$

with equality if and only if $M$ is totally umbilic.

Also,

i.e

$$
\widetilde{\operatorname{div}}((\widetilde{\operatorname{div} \xi}) \xi)=\widetilde{g}(\widetilde{\nabla} \widetilde{\operatorname{div}} \xi, \xi)+(\widetilde{\operatorname{div}} \xi)^{2}
$$

$$
-\widetilde{\operatorname{div}}(H \xi)=-\xi(H)+H^{2}
$$

In [18] we established (2.6), that is $\overline{\operatorname{Ric}}(\xi)=\xi(H)+\tau(\xi) H-\|\stackrel{\star}{A} \xi\|^{2}$. Assume that there is a constant $\lambda$ such that $(\tau(\xi)+\lambda) H \leq 0$. Then

$$
H^{2} \geq \overline{\operatorname{Ric}}(\xi)+\lambda H-\widetilde{\operatorname{div}}(H \xi)
$$

Combining (6.1) and (6.3), we get

$$
\overline{\operatorname{Ric}}(\xi)+\lambda H-\widetilde{\operatorname{div}}(H \xi) \leq H^{2} \leq(n-2)\|\stackrel{\star}{A} \xi\|^{2} .
$$

The equality case in the upper bound (resp. in the lower bound) is attained if and only if $M$ is totally umbilic (resp. $M$ is totally geodesic). Recall from (2.5) that $H=-\widetilde{\operatorname{div}} \xi$. Hence if $M$ is compact without boundary and orientable, by the divergence theorem it holds

$$
\int_{M} \overline{\operatorname{Ric}}(\xi) d M \leq \mathcal{W}(M, \zeta) \leq(n-2) \int_{M}\left\|\stackrel{\star}{A}_{\xi}\right\|^{2} d M .
$$

The equality case in the upper bound is equivalent to $\int_{M}\left(H^{2}-(n-2)\left\|\stackrel{\star}{A}_{\xi}\right\|^{2}\right) d M=0$ and by use of (6.1) this means that $H^{2}=(n-2)\left\|\stackrel{\star}{A}_{\xi}\right\|^{2}$ and $M$ is totally umbilic. For the lower bound, the equality case reads

$$
\int_{M}\left(\widetilde{\operatorname{div}}(H \xi)+\tau(\xi) H-\left\|\stackrel{\star}{A}_{\xi}\right\|^{2}\right) d M=\int_{M}\left(\tau(\xi) H-\left\|\stackrel{\star}{A}_{\xi}\right\|^{2}\right) d M=0=\int_{M}-\lambda H d M
$$

Hence,

$$
\int_{M}\left((\tau(\xi)+\lambda) H-\left\|\stackrel{\star}{A}_{\xi}\right\|^{2}\right) d M=0
$$

As $(\tau(\xi)+\lambda) H \leq 0$ it follows that $(\tau(\xi)+\lambda) H-\left\|\stackrel{\star}{A}_{\xi}\right\|^{2}=0$ and $\|\stackrel{\star}{A} \xi\|^{2}=0$ which means that $M$ is totally geodesic. Thus, we can state.

Theorem 6.1. Let $(M, \zeta)$ be a normalized orientable compact (without boundary) null hypersurface. Suppose there existe a constant $\lambda$ such that $(\tau(\xi)+\lambda) H \leq 0$. Then, the Willmore action has the following bounds:

$$
\int_{M} \overline{\operatorname{Ric}}(\xi) d M \leq \mathcal{W}(M, \zeta) \leq(n-2) \int_{M}\|\stackrel{\star}{A} \xi\|^{2} d M .
$$

The equality case in the upper bound (resp. in the lower bound) is attained if and only if $M$ is totally umbilic (resp. M is totally geodesic).

Remark 6.2. Suppose $M$ is a normalized orientable compact (without boundary) null hypersurface. If H is constant then it vanishes identically. Indeed, from equality in (6.2), it holds by use of the divergence theorem,

$$
0=\int_{M} \xi(H) d M=\int_{M} H^{2} d M \quad \text { which shows that } H=0 .
$$




\subsection{The Euler equation in GRW spacetimes}

In a recent work [29], we pointed out the fact that in Lorentzian manifolds with a closed timelike vector field, there is no compact simply connected null hypersurfaces. But GRW spacetimes do admit such vector fields, (the Chen's ones). So, considering the Willmore problem for null hypersurfaces in GRW spacetimes, we restrict to the family of normalized orientable null hypersurfaces $(M, \zeta)$ for which the mean curvature $H$ has compactly supported variations and for critical points of the Willmore action (the Willmore null hypersurfaces) we apply standard techniques of the calculus of variations.

Let us consider on $\bar{M}$ the frame field $\left(\partial_{t}, \xi, \partial_{u^{2}}, \ldots, \partial_{u^{n-1}}\right)$ with $\left(\partial_{u^{i}}\right)_{2 \leq i \leq n-1}$ tangent to the screen structure $\mathscr{S}(\zeta)$, in which

$$
\bar{g}_{\alpha \beta} \simeq\left(\begin{array}{ccccc}
-1 & \frac{1}{f} & 0 & \ldots & 0 \\
\frac{1}{f} & 0 & & & \\
0 & 0 & & \\
\vdots & \vdots & f^{2}(t) g_{F_{i j}} & \\
0 & 0 & &
\end{array}\right) \quad \text { and } \widetilde{g}_{a b} \simeq \widetilde{G}=\left(\begin{array}{cccc}
1 & 0 & \ldots & 0 \\
0 & & & \\
\vdots & f^{2}(t) g_{F_{i j}} & \\
0 & & &
\end{array}\right) \text {. }
$$

Hence

$$
\operatorname{det} \bar{g}_{\alpha \beta}=-\frac{1}{f^{2}} \operatorname{det} \widetilde{g}_{a b}
$$

Let $v$ be a null coordinate with $\xi=\partial_{v}$. We have

$$
d \bar{g}=\sqrt{-\operatorname{det} \bar{g}_{\alpha \beta}} d t \wedge d v \wedge d u^{2} \cdots \wedge d u^{n-1}
$$

and

$$
\begin{aligned}
d M=i_{\zeta} d \bar{g} & =f(t) \sqrt{-\operatorname{det} \bar{g}_{\alpha \beta}} d v \wedge d u^{2} \cdots \wedge d u^{n-1} \\
& =f(t) \sqrt{\frac{1}{f^{2}} \operatorname{det} \widetilde{g}_{a b}} d v \wedge d u^{2} \cdots \wedge d u^{n-1} \\
& =\sqrt{\operatorname{det} \widetilde{g}_{a b}} d v \wedge d u^{2} \cdots \wedge d u^{n-1} \\
& =d \bar{g}
\end{aligned}
$$

Hence

$$
d M=d \bar{g}
$$

It follows that

$$
\mathcal{W}(M, \zeta)=\int_{M} H^{2} d M=\int_{M}(\widetilde{\operatorname{div}} \xi)^{2} d \widetilde{g} .
$$

Let $p=(t, x) \in I \times_{f} F$ be a generic point on the null hypersurface. We have $x=x\left(u^{1}, \ldots, u^{n-1}\right)$ where $\left(u^{1}, \ldots, u^{n-1}\right)$ denotes coordinates on the fiber $F$. Then $p=p\left(t, u^{1}, \ldots, u^{n-1}\right)$. Now consider a variation of the null hypersurface in the normal direction $\xi$ given by

$$
\bar{p}\left(t, u^{1}, \ldots, u^{n-1}, s\right)=\exp _{\left(t, u^{1}, \ldots, u^{n-1}\right)}\left(s \phi\left(t, u^{1}, \ldots, u^{n-1}\right) \xi\right)
$$

where $\phi$ is a smooth real-valued function and $s$ is real number in a neighborhood of 0 . We denote by $\delta$ the operator

$$
\delta=\left.\frac{\partial}{\partial s}\right|_{s=0}
$$

Willmore null hypersurfaces are those for which

$$
\delta \int_{M} H^{2} d M=0
$$

The following ranges of indices are in use

$$
\begin{gathered}
\alpha, \beta, \gamma, \ldots,=0,1, \ldots, n-1 \\
a, b, c \ldots,=1, \ldots, n-1 \\
i, j, k \ldots,=2, \ldots, n-1 .
\end{gathered}
$$

Without lost of generality, we may assume $\partial_{i}:=\frac{\partial}{\partial u^{i}} \in \mathscr{S}(\zeta)$. Then at each $p, \operatorname{span}\left\{\left.\partial_{t}\right|_{p},\left.\partial u^{1}\right|_{p}\right\}=T_{p} M^{\perp} \oplus \mathbb{R} \zeta$. 
From (6.4), we have

$$
\delta p=\phi \xi
$$

Then,

$$
\begin{aligned}
& \delta \partial_{\left.i\right|_{p}}=\left(\partial_{i} \phi\right) \xi+\phi \bar{\nabla}_{\partial_{i}} \xi \\
& =\left(\partial_{i} \phi\right) \xi-\phi \stackrel{\star}{A} \xi \partial_{i} \text {. } \\
& \delta \xi_{p}=(\xi \cdot \phi) \xi+\phi \bar{\nabla}_{\xi} \xi \\
& =(\xi \cdot \phi) \xi \text { as } \xi \text { is geodesic. } \\
& \delta \partial_{\left.t\right|_{p}}=\left(\partial_{t} \phi\right) \xi+\phi \bar{\nabla}_{\partial_{t}} \xi \\
& =\left(\partial_{t} \phi\right) \xi+\phi\left(\frac{f^{\prime}(t)}{f^{2}(t)} \partial_{t}+\frac{f^{\prime}(t)}{f(t)} \xi^{F}\right) . \\
& =\left(-\frac{\partial_{t} \phi}{f(t)}+\phi \frac{f^{\prime}(t)}{f^{2}(t)}\right) \partial_{t}+\left(\left(\partial_{t} \phi\right)+\phi \frac{f^{\prime}(t)}{f(t)}\right) \xi^{F}
\end{aligned}
$$

and

$$
\delta \partial_{u^{1} \mid p}=\left(\partial_{u^{1}} \phi\right) \xi+\phi \bar{\nabla}_{\partial_{u^{1}}} \xi
$$

Now, we compute $\widetilde{\delta g}_{a b}$.

$$
\begin{aligned}
\bar{g}_{i j}(s) & =\bar{g}\left(\partial_{i}+s\left(\partial_{i} \phi\right) \xi-s \phi \stackrel{\star}{A}_{\xi} \partial_{i}, \partial_{j}+s\left(\partial_{j} \phi\right) \xi-s \phi \stackrel{\star}{A}_{\xi} \partial_{j}\right) \\
& =\widetilde{g}_{i j}+s\left[-\phi \widetilde{g}\left(\partial_{i}, \stackrel{\star}{A}_{\xi} \partial_{j}\right)-\phi \widetilde{g}\left(\stackrel{\star}{A}_{\xi} \partial_{i}, \partial_{j}\right)\right]+s^{2} \phi^{2} \widetilde{g}\left(\stackrel{\star}{A}_{\xi} \partial_{i}, \stackrel{\star}{A}_{\xi} \partial_{j}\right)
\end{aligned}
$$

Hence

$$
\delta \widetilde{g}_{i j}=-2 \phi B\left(\partial_{i}, \partial_{j}\right)
$$

Also,

$$
\delta \widetilde{g}_{0 i}=\delta \widetilde{g}_{i 0}=\partial_{i} \phi \quad \text { and } \quad \delta \widetilde{g}_{00}=2(\xi \cdot \phi)
$$

Using the relation $\sum_{k} \widetilde{g}^{i k} \widetilde{g}_{k j}=\delta_{j}^{i}$,

$$
\sum_{k} \delta \widetilde{g}^{i k} \widetilde{g}_{k j}+\sum_{k} \widetilde{g}^{i k} \delta \widetilde{g}_{k j}=0
$$

Then,

$$
\begin{aligned}
\sum_{k} \delta \widetilde{g}^{i k} \widetilde{g}_{k j} \stackrel{\stackrel{(6.5)}{=}}{=}-\sum_{k} \widetilde{g}^{i k}\left(-2 \phi B\left(\partial_{k}, \partial_{j}\right)\right) \\
=2 \phi \sum_{l} \widetilde{g}^{i l} B\left(\partial_{l}, \partial_{j}\right)
\end{aligned}
$$

It follows that

$$
\delta \widetilde{g}^{i k}=2 \phi \sum_{l j} \widetilde{g}^{k j} \widetilde{g}^{i l} B\left(\partial_{l}, \partial_{j}\right)
$$

Next, put $B_{i j}:=B\left(\partial_{i}, \partial_{j}\right)$. We compute $\delta B_{i j}$. First, we have

$$
\left\langle\bar{\nabla}_{\partial_{i}} \partial_{j}, \delta \xi\right\rangle=\left\langle\nabla_{\partial_{i}} \partial_{j}+B_{i j} N,(\xi \cdot \phi) \xi\right\rangle=(\xi \cdot \phi) B_{i j}
$$


Also,

$$
\begin{aligned}
\bar{\nabla}_{\partial_{i}(s)} \partial_{j}(s) & =\bar{\nabla}_{\partial_{i}+s\left(\partial_{i} \phi\right) \xi-s \phi \stackrel{\star}{\xi}_{\xi} \partial_{i}}\left(\partial_{j}+s\left(\partial_{j} \phi\right) \xi-s \phi \stackrel{\star}{A}_{\xi} \partial_{j}\right) \\
& =\bar{\nabla}_{\partial_{i}} \partial_{j}+s\left[\bar{\nabla}_{i}\left(\partial_{j} \phi\right) \xi-\bar{\nabla}_{\partial_{i}}\left(\phi \stackrel{\star}{A}_{\xi} \partial_{j}\right)\right]+\text { second order term in } s
\end{aligned}
$$

which gives

$$
\begin{aligned}
\delta \bar{\nabla}_{\partial_{i}} \partial_{j}= & \bar{\nabla}_{i}\left(\partial_{j} \phi\right) \xi-\bar{\nabla}_{\partial_{i}}\left(\phi \stackrel{\star}{A}_{\xi} \partial_{j}\right) \\
= & \left(\partial_{i} \partial_{j} \phi\right) \xi+\left(\partial_{j} \phi\right)\left(-\stackrel{\star}{A}_{\xi} \partial_{i}\right)-\left(\partial_{i} \phi\right) \stackrel{\star}{A_{\xi}} \partial_{j}-\phi\left(\nabla_{\partial_{i}} \stackrel{\star}{A}_{\xi} \partial_{j}\right) \\
& -\phi B\left(\partial_{i} \stackrel{\star}{A} \partial_{j}\right) N+(\partial \phi) \nabla_{\xi} \partial_{j}-\phi \nabla_{\AA_{\xi} \partial_{i}} \partial_{j}-\phi B\left(\stackrel{\star}{A}_{\xi} \partial_{i}, \partial_{j}\right) .
\end{aligned}
$$

It follows that

$$
\left\langle\delta \bar{\nabla}_{\partial_{i}} \partial_{j}, \xi\right\rangle=-2 \phi B\left(\partial_{i} \stackrel{\star}{A}_{\xi} \partial_{j}\right)=-2 \phi \widetilde{g}\left(\stackrel{\star}{A}_{\xi} \partial_{i}, \stackrel{\star}{A}_{\xi} \partial_{j}\right)
$$

From $B_{i j}=\left\langle\bar{\nabla}_{\partial_{i}} \partial_{j}, \xi\right\rangle$ it follows,

$$
\begin{aligned}
\delta B_{i j} & =\left\langle\delta \bar{\nabla}_{\partial_{i}} \partial_{j}, \xi\right\rangle+\left\langle\bar{\nabla}_{\partial_{i}} \partial_{j}, \delta \xi\right\rangle \\
& =-2 \phi\left\langle\stackrel{\star}{A}_{\xi} \partial_{i}, \stackrel{\star}{A}_{\xi} \partial_{j}\right\rangle+(\xi \cdot \phi) B_{i j} .
\end{aligned}
$$

But

$$
H=\sum_{i j} \widetilde{g}^{i j} \widetilde{g}\left(\stackrel{\star}{A}_{\xi} \partial_{i}, \partial_{j}\right)=\sum_{i j} \widetilde{g}^{i j} B_{i j}
$$

Then,

$$
\begin{aligned}
\delta H & =\sum_{i j} \delta \widetilde{g}^{i j} B_{i j}+\sum_{i j} \widetilde{g}^{i j} \delta B_{i j} \\
& =\sum_{i j}\left(2 \phi \sum_{l m} \widetilde{g}^{i l} l^{j m} B_{l m}\right) B_{i j}+\sum_{i j} \widetilde{g}^{i j}\left[-2 \phi \widetilde{g}\left(\stackrel{\star}{A}_{\xi} \partial_{i}, \stackrel{\star}{A}_{\xi} \partial_{j}\right)+(\xi \cdot \phi) B_{i j} \cdot\right] \\
& =2 \phi\left\|\stackrel{\star}{A}_{\xi}\right\|^{2}-2 \phi\left\|\stackrel{\star}{A}_{\xi}\right\|^{2}+(\xi \cdot \phi) H
\end{aligned}
$$

since $\stackrel{\star}{A}_{\xi} \partial_{i}=\widetilde{g}^{l m} B_{i m} \partial_{l}$ and the first term at the right hand side is $2 \phi\left\|\stackrel{\star}{A}_{\xi}\right\|^{2}$. Thus,

$$
\delta H=(\xi \cdot \phi) H .
$$

Now, let $\Omega=\sqrt{\operatorname{det} \widetilde{g}_{a b}}=\sqrt{\operatorname{det} \widetilde{g}_{i j}}$. Then

$$
2 \Omega \frac{\partial \Omega}{\partial s}=\Omega^{2} \operatorname{trace}\left(\widetilde{G}^{-1} \cdot \frac{\partial \widetilde{G}}{\partial s}\right)
$$

i.e

$$
\begin{aligned}
\delta \Omega & =\frac{1}{2} \Omega \operatorname{trace}\left(\widetilde{g}^{i k} \delta \widetilde{g}_{k j}\right) \stackrel{(6.5)}{=} \frac{1}{2} \Omega \operatorname{trace}\left(\widetilde{g}^{i k}\left(-2 \phi B_{k j}\right)\right) \\
& =-\phi \Omega \operatorname{trace} \widetilde{g}^{i k} B_{k j}=-\phi \Omega \widetilde{g}^{i j} B_{i j}=-\phi \Omega H .
\end{aligned}
$$

Finally,

$$
\begin{aligned}
\delta \int_{M} H^{2} d \widetilde{g} & =\int_{M} 2 H \delta H d \bar{g}+\int_{M} H^{2} \delta \widetilde{g} \\
& =\int_{M} 2 H(\xi \cdot \phi) H d \bar{g}+\int_{M} H^{2}(-\phi H) d \widetilde{g}
\end{aligned}
$$

i.e

$$
\delta \int_{M} H^{2} d \bar{g}=\int_{M} H^{2}[2(\xi \cdot \phi)-H \phi] d \widetilde{g} .
$$

Thus the condition that the integral is stationary for all smooth function $\phi$ is $H=0$. Indeed, take $\phi=H$. Then

$$
0=\int_{M} H^{2}\left[2(\xi \cdot H)-H^{2}\right] d \bar{g}=\int_{M}\left[2 H^{2}(\xi \cdot H)-H^{4}\right] d \widetilde{g}
$$




$$
\begin{aligned}
& =\int_{M}\left[\frac{2}{3}\left(\xi \cdot H^{3}\right)-H^{4}\right] d \widetilde{g}=\int_{M}\left[-\frac{2}{3} H^{3} \widetilde{\operatorname{div}} \xi-H^{4}\right] d \widetilde{g} \\
& =\int_{M}\left[\frac{2}{3} H^{4}-H^{4}\right] d \widetilde{g}=-\frac{1}{3} \int_{M} H^{4} d \widetilde{g} .
\end{aligned}
$$

Then we can state the following:

Theorem 6.3. In a generalized Robertson-Walker spacetime, the only Willmore normalized null hypersurfaces $(M, \zeta)$ where $\zeta$ is the closed conformal timelike concircular vector field $f \partial_{t}$ are the maximal ones.

\section{References}

[1] J. Aledo, R. Rubio, J. Salamanca, Complete spacelike hypersurfaces in generalized Robertson-Walker and the null convergence condition: Calabi-Bernstein problem, RACSAM Rev. R. Acad. Cienc. Exactas F'1s. Nat. Ser. A Mat., doi: 10.1007/s13398-016-0277-3 (2016).

[2] L. J. Alias, P. Mira, On the Calabi-Bernstein theorem for maximal hypersurfaces in the Lorentz-Minkowski space, Publ. R. Soc. Mat. Esp., 4 (2003), $23-55$.

[3] L. J. Alias, A. Romero, M. Sánchez, Uniqueness of complete spacelike hypersurfaces of constant mean curvature in Generalized Robertson-Walker spacetimes, Gen. Relativity Gravitation, 27 (1995), 71-84

4] R. Bartnik, Existence of maximal surfaces in asymptotically flat spacetimes, Comm. Math. Phys., 94 (1984), 155-175.

[5] D. Brill, F. Flaherty, Isolated maximal surfaces in spacetime, Comm. Math. Phys., 50 (1976), 157-165.

[6] S. Y. Cheng, S. T. Yau, Maximal spacelike hypersurfaces in the Lorentz-Minkowski spaces, Ann. of Math. (2), 104(3) (1976), 407-419.

[7] Y. Choquet-Bruhat, A. Fischer, J. Marsden, Isolated Gravitating Systems in General Relativity, Italian, $1979,396-456$.

[8] Y. Choquet-Bruhat, Quelques propriétés des sous-variétés maximales d'une variété lorentzienne, C. R. Math. Acad. Sci. Paris, Serie A, 281 (1975), $577-580$.

[9] H. F. de Lima, U. L. Parente, On the geometry of maximal spacelike hypersurfaces immersed in a generalized Robertson-Walker spacetime, Ann. Mat. Pura Appl. 192 (2013), 649-663.

[10] C. A. Mantica, L. G. Molinardi, Generalized Robertson-Walker spacetimes - A survey, Int. J. Geom. Methods Mod. Phys., 14 (2017), 1730001 (27 pages) doi: 10.1142/S021988781730001X

[11] C. A. Mantica, L. G. Molinardi, On the Weyl and Ricci tensors of deneralized Robertson-Walker spacetimes, J. Math. Phys., 57, 102502 (2016), doi: $10.1063 / 1.4965714$.

[12] J. E. Marsden, F. J. Tipler, Maximal hypersurfaces and foliations of constant mean curvature in general relativity, Phys. Rep., 66(3) (1980), 109-139.

[13] A. Romero, R. Rubio, J. J. Salamanca, Uniqueness of complete maximal hypersurfaces in spatially parabolic generalized Robertson-Walker spacetimes, Classical Quantum Gravity, 30 (2013), 115007.

[14] M. Barros, Free elasticae and Willmore tori in warped product spaces, Glasg. Math. J., 40 (1998), 265-272.

[15] M. Barros, A geometric algorithm to construct new solitons in the O(3) nonlinear sigma model, Phys. Lett. B, 553(3-4) (2003), 325-331.

[16] M. Barros, O. J. Garay, A. D. Singer, Elasticae with constant slant in the complex projective plane and new examples of Willmore tori in five spheres, Tohoku Math. J. (2), 51(2) (1999), 177-192.

[17] J. L. Weiner, On the problem of Chen, Willmore, et al., Indiana Univ. Math.1 J., 27 (1978), 19-35.

[18] C. Atindogbe, H. Fotsing Tetsing, Newton transformations on null hypersurfaces, Commun. Math., 23 (2015), 57-83.

[19] C. Atindogbe, Scalar curvature on lightlike hypersurfaces, Appl. Sci., 11 (2009), 9-18.

[20] K. L. Duggal, A. Bejancu, Lightlike Submanifolds of Semi-Riemannian Manifolds and Applications, Kluwer Academic, 364, Dordrecht, 1996.

[21] K. L. Duggal B. Sahin, Differential Geometry of Lightlike Submanifolds, Birkhaüser Verlag AG, Basel, 2010.

[22] G. J. Galloway, Maximum principles for null hypersurfaces and null splitting theorems, Annes de 1'institut Henri Poincaré, 1, (2000), 543-567.

[23] M. Gutiérrez, B. Olea, Induced Riemannian structures on null hypersurfaces, Math. Nachr., (2015), 1-18, doi: https://doi.org/10.1002/mana.201400355.

[24] M. Gutiérrez, B. Olea, Totally umbilic null hypersurfaces in generalized Robertson-Walker spaces Differ. Geom. Appl., 42 (2015), 15-30.

[25] B. Y. Chen, A simple characterization of generalized Robertson-Walker spacetimes, Gen. Relativity Gravitation, 46:1833 (2014), doi: 10.1007/s10714014-1833-9.

[26] A. Fialkow, Conformals geodesics, Trans. Amer. Math. Soc., 45(3) (1939), 443-473.

[27] K. L. Duggal, On scalar curvature in lightlike geometry, J. Geom. Phys., 57 (2007), 473-481.

[28] H. K. El-Sayied, S. Shenawy, N. Syed, On symmetries of generalized Robertson-Walker spacetimes and applications, J. Dyn. Syst. Geom. Theor., 15(1) (2017), 51-69.

[29] C. Atindogbe M. Gutierrez, R. Hounnonkpè, New properties on normalized null hypersurfaces, Mediterranean J. Math. Appl., 15:166 (2018), doi: 10.1007/s00009-018-1210-0 